\title{
Effects of Variable Viscosity and Thermal Conductivity on Micropolar Fluid Flow Due to a Stretching Cylinder in Presence of Magnetic Field
}

\author{
Surajit Dutta \\ Department of Mathematics \\ C.N.B. College, Bokakhat, Assam, India \\ Corresponding author: surajitdutta505@gmail.com \\ Bishwaram Sharma \\ Department of Mathematics \\ Dibrugarh University, Dibrugarh, Assam, India \\ E-mail: brsharma@dibru.ac.in \\ Gopal Chandra Hazarika \\ Department of Mathematics \\ Dibrugarh University, Dibrugarh, Assam, India \\ E-mail: gchazarika@dibru.ac.in \\ (Received January 1, 2019; Accepted March 30, 2019)
}

\begin{abstract}
In the presence of magnetic field, steady flow of a micropolar fluid due to a stretching cylinder is studied. Viscosity and thermal conductivity are assumed to be inverse linear functions of temperature. The governing partial differential equations are converted into ordinary differential equations using suitable similarity transformations and then solved by fourth order Runge-Kutta shooting method and developing Matlab programme. The graphs show the effects of different parameters and the skin friction coefficient and Nusselt numbers are shown in tabular form.
\end{abstract}

Keywords- Micropolar fluid, Stretching cylinder, Variable viscosity, Thermal conductivity.

\section{Introduction}

From the very beginning of the introduction of micropolar fluid theory by Eringen $(1964,1966$, 1972), it becomes an attractive part of so many researchers. This theory is theoretical, for which attracts Mathematicians as well as practical attracting Physicists and Engineers. The micropolar fluid theory is the most appropriate theory among various theories to study the behaviour of nonNewtonian fluids. There are so many fluids which are industrially important and showing nonNewtonian behaviours. Biological fluids, multiphase mixtures, food products are some such fluids. Boundary layer theory for micropolar fluid was studied by Peddieson and McNitt (1970). Guram and Smith (1980) investigated the stagnation flow of micropolar fluids with strong and weak interactions. Time dependent slip flow of a micropolar fluid between two parallel plates through a state space approach was examined by Slayi et al. (2016). Sheri and Shamshuddin (2015) studied heat and mass transfer on the MHD (magnetohydrodynamic) flow of micropolar fluid in the presence of viscous dissipation and chemical reaction. MHD unsteady flow and heat transfer of micropolar fluid through the porous channel with expanding or contracting walls were investigated by Asia et al. (2016).

The problem of MHD flow and heat transfer over a stretching surface have been studied by so many researchers during the last few decades as it is applicable in various industries. For 
International Journal of Mathematical, Engineering and Management Sciences

Vol. 4, No. 3, 745-760, 2019

https://dx.doi.org/10.33889/IJMEMS.2019.4.3-058

example, in the extrusion of a polymer sheet, the properties of the final product considerably depend on the rate of cooling. MHD flow and heat transfer of a micropolar fluid over a stretching surface with heat generation (absorption) and slip velocity was studied by Mahmoud and Waheed (2012). Hazarika and Phukan (2016) investigated the effects of variable viscosity and thermal conductivity on magnetohydrodynamic free convection flow of a micropolar fluid past a stretching plate through a porous medium with radiation, heat generation, and Joule dissipation. Effects of variable viscosity and thermal conductivity on magnetohydrodynamics mixed convective flow over a stretching surface with radiation have been studied by Hazarika and Hazarika (2015). Ishak et al. (2008) examined heat transfer over a stretching surface with variable heat flux in micropolar fluids. Convective heat transfer in an electrically conducting fluid at a stretching surface in the uniform free stream was studied by Vajravelu and Hadjinicalaou (1997).

Effects of variable viscosity and thermal conductivity on unsteady micropolar fluid about a permeable cylinder under moving boundaries have been studied by Baruah and Hazarika (2017). Hayat et al. (2016) investigated magnetohydrodynamics flow by a stretching cylinder with Newtonian heating and homogeneous heterogeneous reactions.

In this study, we have extended the work of Nazir and Shafique (2015) and analyse the effects of variable viscosity and thermal conductivity on micropolar fluid flow due to a stretching cylinder in the presence of magnetic field. In most of the earlier studies, physical properties were assumed to be constant. Here we consider the viscosity and thermal conductivity as inverse linear functions of temperature. The governing partial differential equations are reduced to ordinary differential equations using similarity transformations and then solved numerically by fourth order Runge-Kutta shooting method under prescribed boundary conditions.

\section{Mathematical Formulation}

We consider the steady viscous incompressible micropolar fluid flow due to a stretching cylinder in the presence of magnetic field. The cylindrical coordinate system $(r, \theta, z)$ is considered and the cylinder is described with radius $r=a$. Here, the material constants of the micropolar fluid are assumed to be independent of position and neglect body force and body couple. Due to axial symmetry in the prescribed cylindrical coordinate system only two components are there. The velocity components are given by $(u(r, z), 0, w(r, z))$ and micro rotation components are given by $(0, N(r, z), 0)$. Under these conditions the governing equations in the cylindrical coordinate system are as below:

$$
\begin{aligned}
& \begin{aligned}
& \frac{\partial u}{\partial r}+\frac{u}{r}+\frac{\partial w}{\partial z}=0 \\
& \begin{aligned}
\rho\left(u \frac{\partial u}{\partial r}+w \frac{\partial u}{\partial z}\right)=-\frac{\partial p}{\partial r}+(\mu+\kappa)\left(\frac{\partial^{2} u}{\partial r^{2}}+\frac{1}{r} \frac{\partial u}{\partial r}+\frac{\partial^{2} u}{\partial z^{2}}-\frac{u}{r^{2}}\right) & +2 \frac{\partial \mu}{\partial r} \frac{\partial u}{\partial r}+\frac{\partial \mu}{\partial z}\left(\frac{\partial u}{\partial z}+\frac{\partial w}{\partial r}\right) \\
& -\kappa \frac{\partial N}{\partial z}-\sigma B_{0}^{2} u
\end{aligned} \\
& \begin{aligned}
\rho\left(u \frac{\partial w}{\partial r}+w \frac{\partial w}{\partial z}\right)=-\frac{\partial p}{\partial z}+(\mu+\kappa)\left(\frac{\partial^{2} w}{\partial r^{2}}+\frac{1}{r} \frac{\partial w}{\partial r}+\frac{\partial^{2} w}{\partial z^{2}}\right)+2 \frac{\partial \mu}{\partial z} \frac{\partial w}{\partial z}+\frac{\partial \mu}{\partial r}\left(\frac{\partial u}{\partial z}+\frac{\partial w}{\partial r}\right) \\
-\kappa\left(\frac{N}{r}+\frac{\partial N}{\partial r}\right)-\sigma B_{0}^{2} w
\end{aligned} \\
& \rho j\left(u \frac{\partial N}{\partial r}+w \frac{\partial N}{\partial z}+\frac{u N}{r}\right)=\gamma\left(\frac{\partial^{2} N}{\partial r^{2}}+\frac{1}{r} \frac{\partial N}{\partial r}+\frac{\partial^{2} N}{\partial z^{2}}-\frac{N}{r^{2}}\right)+\kappa\left(\frac{\partial u}{\partial z}-\frac{\partial w}{\partial r}\right)-2 \kappa N
\end{aligned}
\end{aligned}
$$


International Journal of Mathematical, Engineering and Management Sciences

Vol. 4, No. 3, 745-760, 2019

https://dx.doi.org/10.33889/IJMEMS.2019.4.3-058

$\rho c_{p}\left(u \frac{\partial T}{\partial r}+w \frac{\partial T}{\partial z}\right)=\lambda\left(\frac{\partial^{2} T}{\partial r^{2}}+\frac{1}{r} \frac{\partial T}{\partial r}+\frac{\partial^{2} T}{\partial z^{2}}\right)+2 \frac{\partial \lambda}{\partial r} \frac{\partial T}{\partial r}+\frac{\partial \lambda}{\partial z} \frac{\partial T}{\partial z}$

Boundary conditions are

$$
\left.\begin{array}{cl}
u=0, w=2 k z, N=0, T=T_{w} & \text { at } r=a \\
u \rightarrow 0, N \rightarrow 0, T \rightarrow T_{\infty} & \text { as } r \rightarrow \infty
\end{array}\right\}
$$

where $\mu$ is the co-efficient of dynamic viscosity, $\kappa$ is the vortex viscosity, $\rho$ is the density, $g_{0}$ is the acceleration due to gravity, $N$ is the angular velocity, $T$ is the fluid temperature, $\sigma$ is the electrical conductivity, $B_{0}$ is the magnetic field intensity, $\gamma$ is the micro rotation viscosity, $j$ is the micro rotation density, $\lambda$ is the thermal conductivity, $c_{p}$ is the specific heat at constant pressure, $P$ is the pressure. $T_{w}$ represents the constant temperature at the surface of the cylinder and $T_{\infty}$ represent the temperature far away from the surface where $T_{w}>T_{\infty}$.

According to Lai and Kulacki (1990)

$$
\frac{1}{\mu}=\frac{1}{\mu_{\infty}}\left[1+\delta\left(T-T_{\infty}\right)\right], \text { or } \frac{1}{\mu}=a\left(T-T_{r}\right)
$$

where $T_{r}$ is transformed reference temperature corresponding to viscosity parameter, $\mu$ is the viscosity, $\mu_{\infty}$ is the viscosity of the ambient fluid, $a$ and $T_{\infty}$ are constants whose values are depend upon the reference state and thermal property of the fluid.

According to Khound and Hazarika (2000)

$$
\frac{1}{\lambda}=\frac{1}{\lambda_{\infty}}\left[1+\xi\left(T-T_{\infty}\right)\right], \text { or } \frac{1}{\lambda}=b\left(T-T_{c}\right)
$$

where $\lambda_{\infty}$ is the thermal conductivity of the ambient fluid, $b$ and $T_{c}$ are constants depending on the reference state and thermal properties of the fluid.

We introduce the following similarity transformations and parameters:

$$
\left.\begin{array}{c}
u=-\frac{k a f(\eta)}{\sqrt{\eta}}, \quad w=2 k f^{\prime}(\eta) z, \quad N=-\frac{4 k r z}{a^{2}} h(\eta) \\
\eta=\left(\frac{r}{a}\right)^{2}, \quad \theta=\frac{T-T_{\infty}}{T_{w}-T_{\infty}}
\end{array}\right\}
$$

Using the above transformations continuity equation (1) is identically satisfied and equations (2) - (4) becomes 
International Journal of Mathematical, Engineering and Management Sciences

Vol. 4, No. 3, 745-760, 2019

https://dx.doi.org/10.33889/IJMEMS.2019.4.3-058

$$
\begin{aligned}
& \begin{aligned}
&\left(\frac{\theta_{r}}{\theta-\theta_{r}}-C_{1}\right) \eta f^{\prime v} \\
&= f^{\prime \prime \prime}\left[\frac{2 \theta_{r}}{\left(\theta-\theta_{r}\right)^{2}} \eta \theta^{\prime}-2\left(\frac{\theta_{r}}{\theta-\theta_{r}}-C_{1}\right)+R e f\right] \\
&+\left[\frac{2 \theta_{r} \theta^{\prime}}{\left(\theta-\theta_{r}\right)^{2}}-\frac{2 \theta_{r}}{\left(\theta-\theta_{r}\right)^{3}} \eta\left(\theta^{\prime}\right)^{2}-R e f^{\prime}-M\right] f^{\prime \prime}+\frac{\theta_{r}}{\left(\theta-\theta_{r}\right)^{2}} \eta f^{\prime \prime} \theta^{\prime \prime} \\
&+2 C_{1} h^{\prime}+C_{1} \eta h^{\prime \prime}
\end{aligned} \\
& \eta h^{\prime \prime}=-\left(2+C_{3} f\right) h^{\prime}+\left(C_{3} f^{\prime}-\frac{C_{3}}{\eta} f+2 C_{2}\right) h-C_{2} f^{\prime \prime} \\
& \left(\frac{\theta_{c}}{\theta-\theta_{c}}\right) \eta \theta^{\prime \prime}= \\
& R e P_{r} f \theta^{\prime}-\frac{\theta_{c} \theta^{\prime}}{\theta-\theta_{c}}+\frac{2 \theta_{c} \eta}{\left(\theta-\theta_{c}\right)^{2}}\left(\theta^{\prime}\right)^{2}
\end{aligned}
$$

where $\theta_{r}$ and $\theta_{c}$ are the dimensionless reference temperatures corresponding to viscosity and thermal conductivity parameter respectively.

The boundary conditions (6) become

$$
\begin{aligned}
& \left.f=0, \quad f^{\prime}=1, \quad \theta=1, \quad h=0, f^{\prime \prime \prime}=R e+C_{1} h^{\prime}-f^{\prime \prime} \quad \text { at } \eta=1\right\}
\end{aligned}
$$

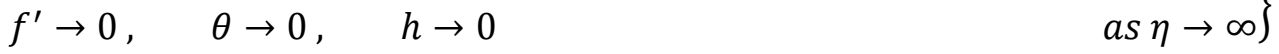

The dimensionless parameters are defined here as:

$C_{1}=\frac{\kappa}{\mu_{\infty}}, C_{2}=\frac{a^{2} \kappa}{4 \gamma}$ and $C_{3}=\frac{a^{2} \kappa \rho j}{2 \gamma}$ are the micropolar parameters; $M=\frac{\sigma a^{2} B_{0}^{2}}{4 \mu_{\infty}}$ is the Hartmann number; $P_{r}=\frac{\mu_{\infty} C_{p}}{\lambda_{\infty}}$ is the Prandtl number.

In this problem, important physical quantities are the skin friction coefficient $c_{f}$ and the local Nusselt number $\mathrm{Nu}$ which indicate physically the shear stress of the surface and the rate of heat transfer respectively. These are defined as

$$
c_{f}=\frac{2 \tau_{w}}{\rho w^{2}}
$$

where $\tau_{w}$ is the shear stress of the surface given by

$$
\tau_{w}=\left[(\mu+\kappa) \frac{\partial w}{\partial r}\right]_{r=a}
$$

and

$$
N u=\frac{z q_{w}}{\lambda_{\infty}\left(T_{w}-T_{\infty}\right)}
$$

where $q_{w}$ is the heat transfer from the surface given by

$$
q_{w}=-\lambda\left[\frac{\partial T}{\partial r}\right]_{r=a} .
$$


International Journal of Mathematical, Engineering and Management Sciences

Vol. 4, No. 3, 745-760, 2019

https://dx.doi.org/10.33889/IJMEMS.2019.4.3-058

After some simple steps we get

$\frac{z c_{f} R e}{a}=\left[C_{1}-\frac{\theta_{r}}{1-\theta_{r}}\right] f^{\prime \prime}(1)$.

and

$$
\frac{a N u}{2 z}=\frac{\theta_{c}}{1-\theta_{c}} \theta^{\prime}(1)
$$

\section{Results and Discussion}

The system of differential equations (10)-(12) together with the boundary conditions (13) are solved numerically by using fourth order Runge-Kutta shooting method and developing codes in MATLAB. The results are shown graphically for different parameters involved in the equations. The numerical values of different parameters are taken as $h=0.02, M=0.1, \theta_{c}=-12, \theta_{r}=$ $-8, C_{1}=0.1, C_{2}=0.1, C_{3}=0.25, P r=0.72, R e=10$ and $N=0.5$ unless otherwise stated. Figures (1) - (4) illustrates the effects of velocity distribution with $C_{1}, C_{2}, \theta_{r}$ and $\theta_{c}$. It is observed that velocity increase for the increase of micropolar parameters $C_{1}$ and $C_{2}$, viscosity parameter $\theta_{r}$ and thermal conductivity parameter $\theta_{c}$.

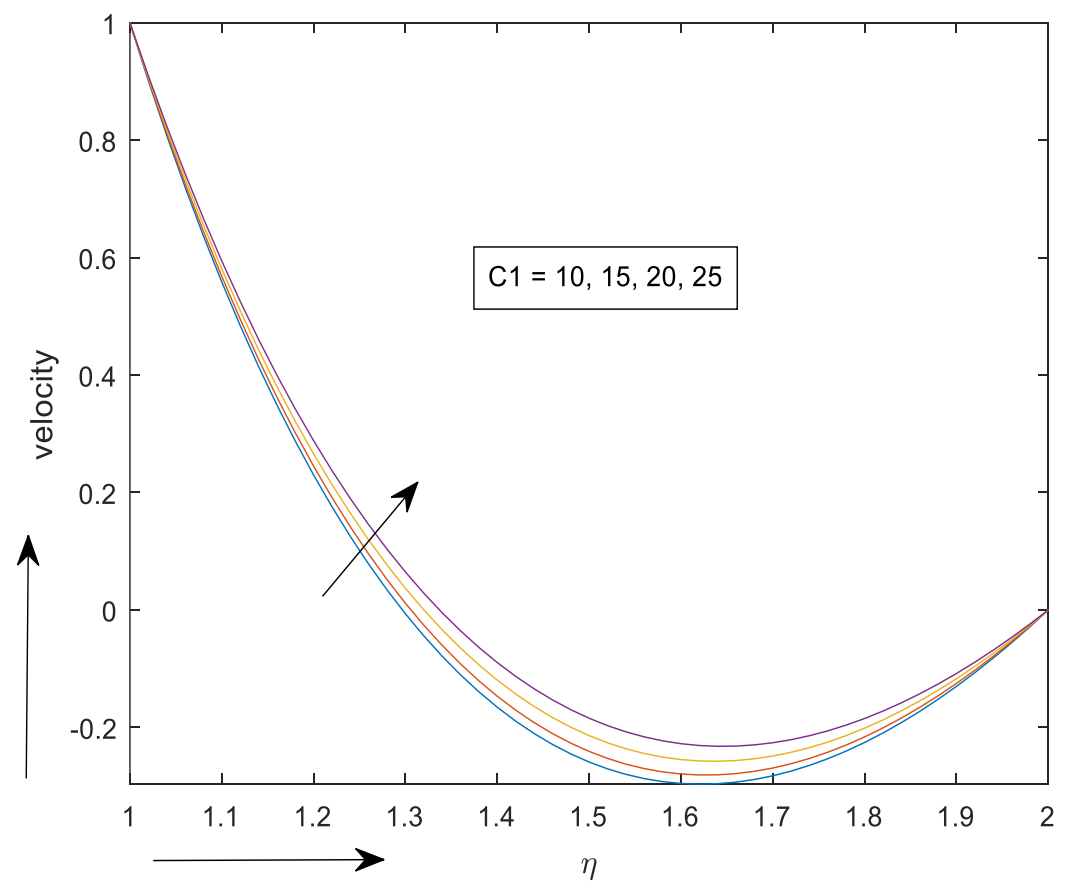

Figure 1 . Velocity profile for different values of $C_{1}$ 
International Journal of Mathematical, Engineering and Management Sciences

Vol. 4, No. 3, 745-760, 2019

https://dx.doi.org/10.33889/IJMEMS.2019.4.3-058

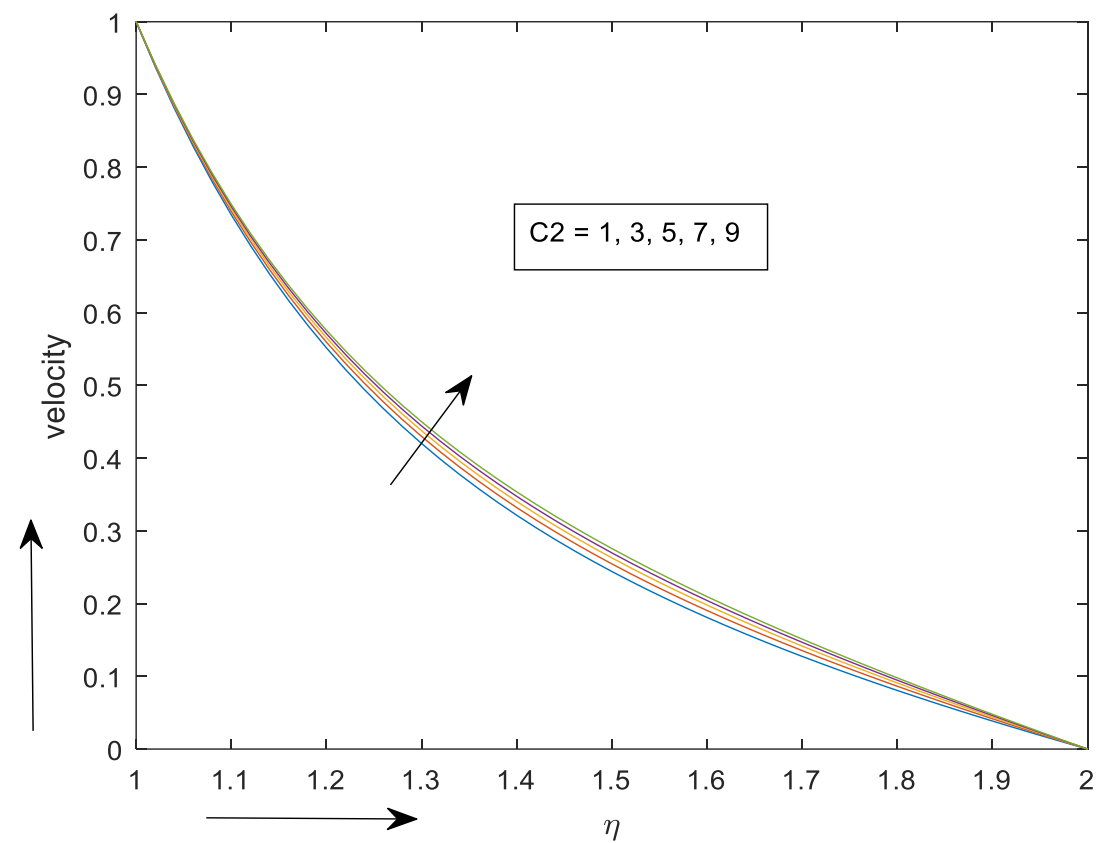

Figure 2. Velocity profile for different values of $C_{2}$

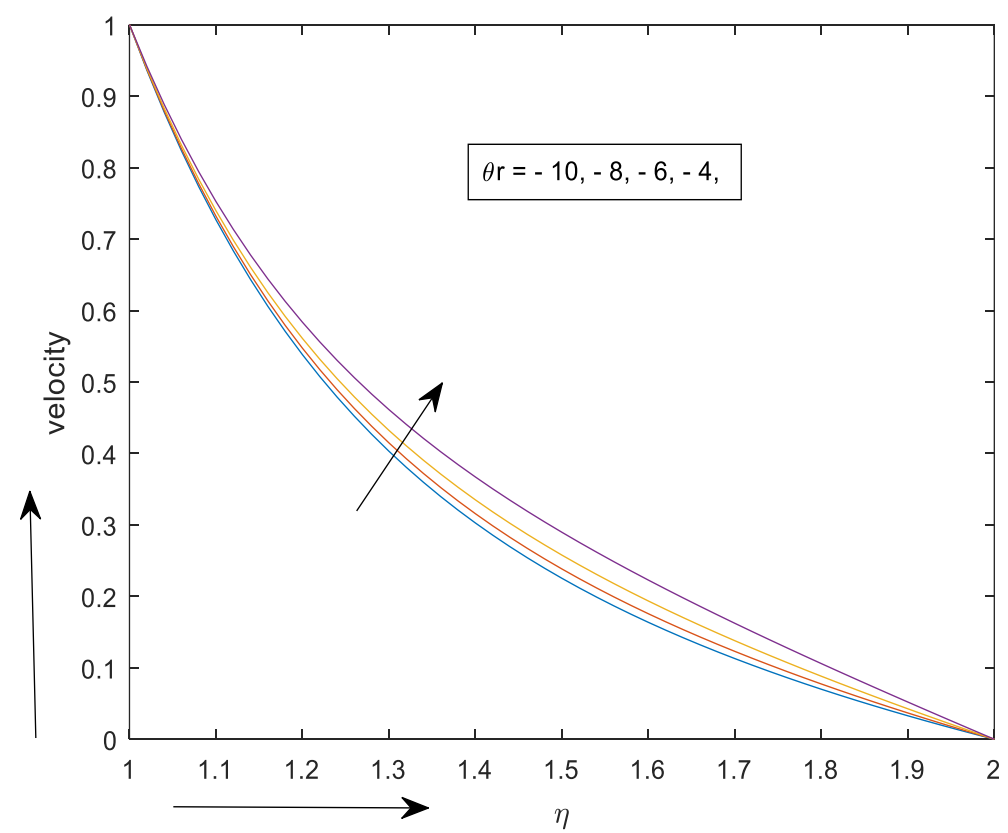

Figure 3. Velocity profile for different values of $\theta_{r}$ 
International Journal of Mathematical, Engineering and Management Sciences

Vol. 4, No. 3, 745-760, 2019

https://dx.doi.org/10.33889/IJMEMS.2019.4.3-058

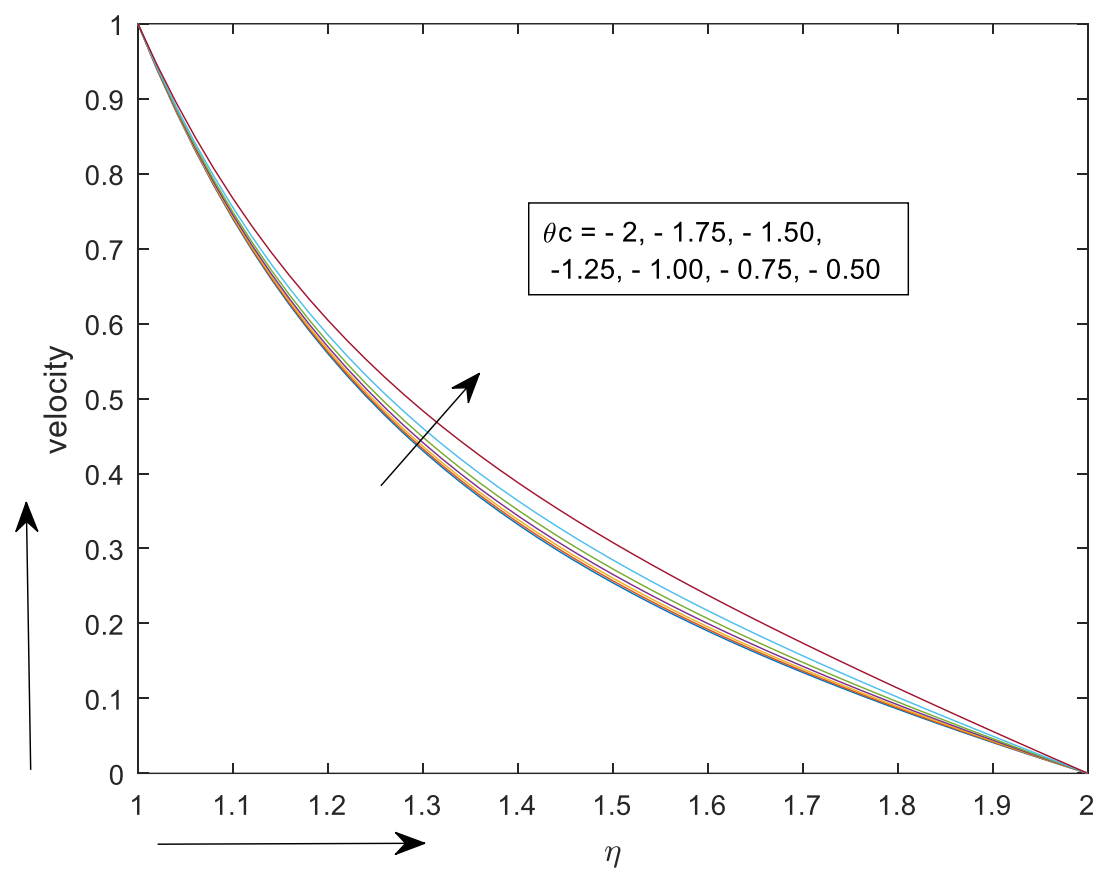

Figure 4. Velocity profile for different values of $\theta_{c}$

The temperature profile for parameters $M, C_{1}, \theta_{r}$ and $\theta_{c}$ are presented in the Figures 5 to 8 . From these figures, it is observed that temperature decreases for the increase of viscosity parameter $\theta_{r}$ and thermal conductivity parameter $\theta_{c}$ but temperature increases for the increase of magnetic parameter $M$ and micropolar parameter $C_{1}$. 
International Journal of Mathematical, Engineering and Management Sciences

Vol. 4, No. 3, 745-760, 2019

https://dx.doi.org/10.33889/IJMEMS.2019.4.3-058

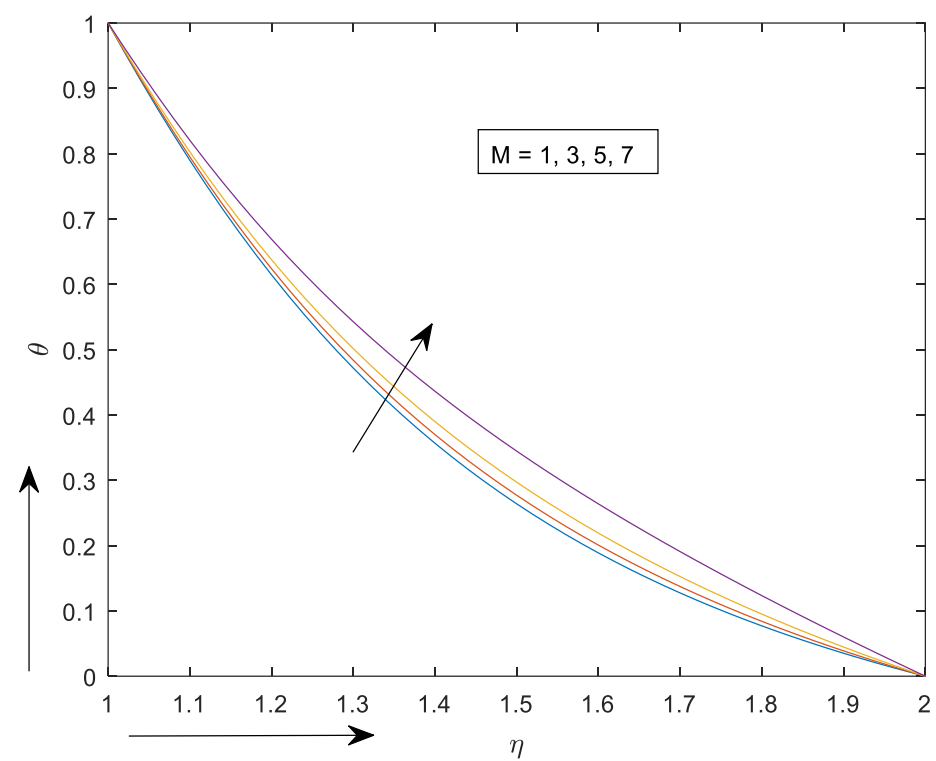

Figure 5. Temperature profile for different values of $M$

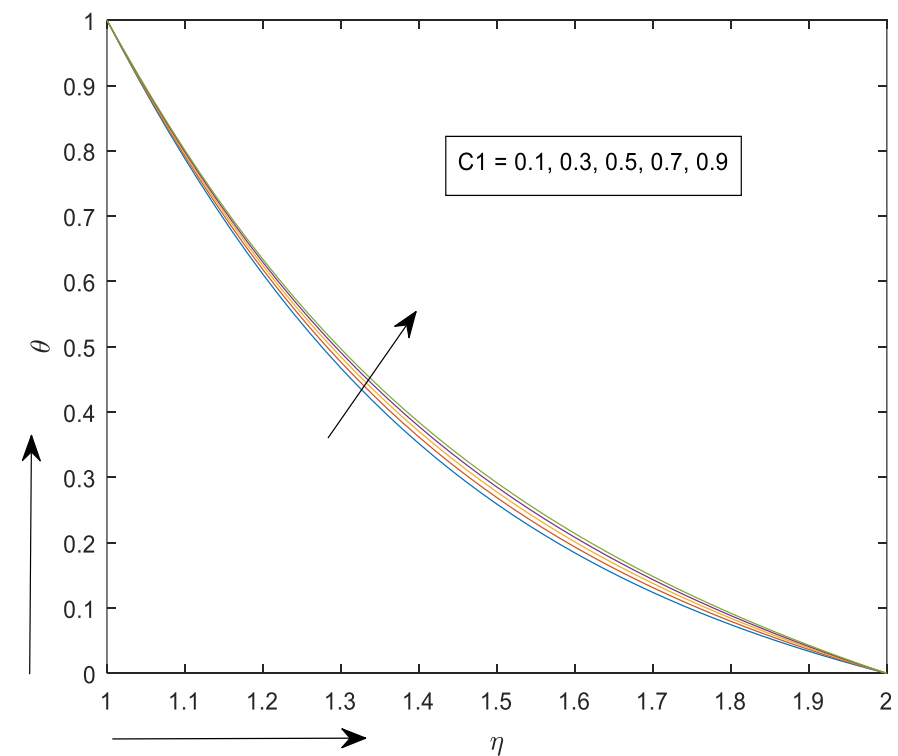

Figure 6. Temperature profile for different values of $C_{1}$ 
International Journal of Mathematical, Engineering and Management Sciences

Vol. 4, No. 3, 745-760, 2019

https://dx.doi.org/10.33889/IJMEMS.2019.4.3-058

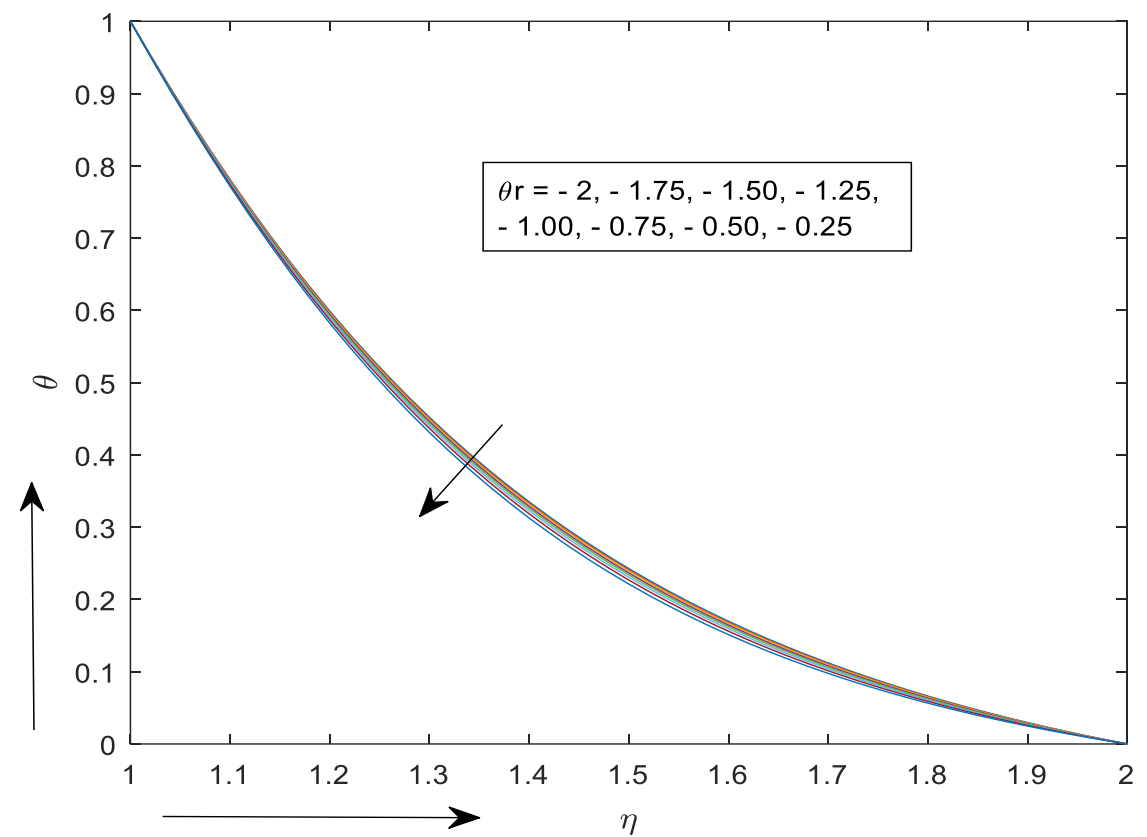

Figure 7. Temperature profile for different values of $\theta_{r}$

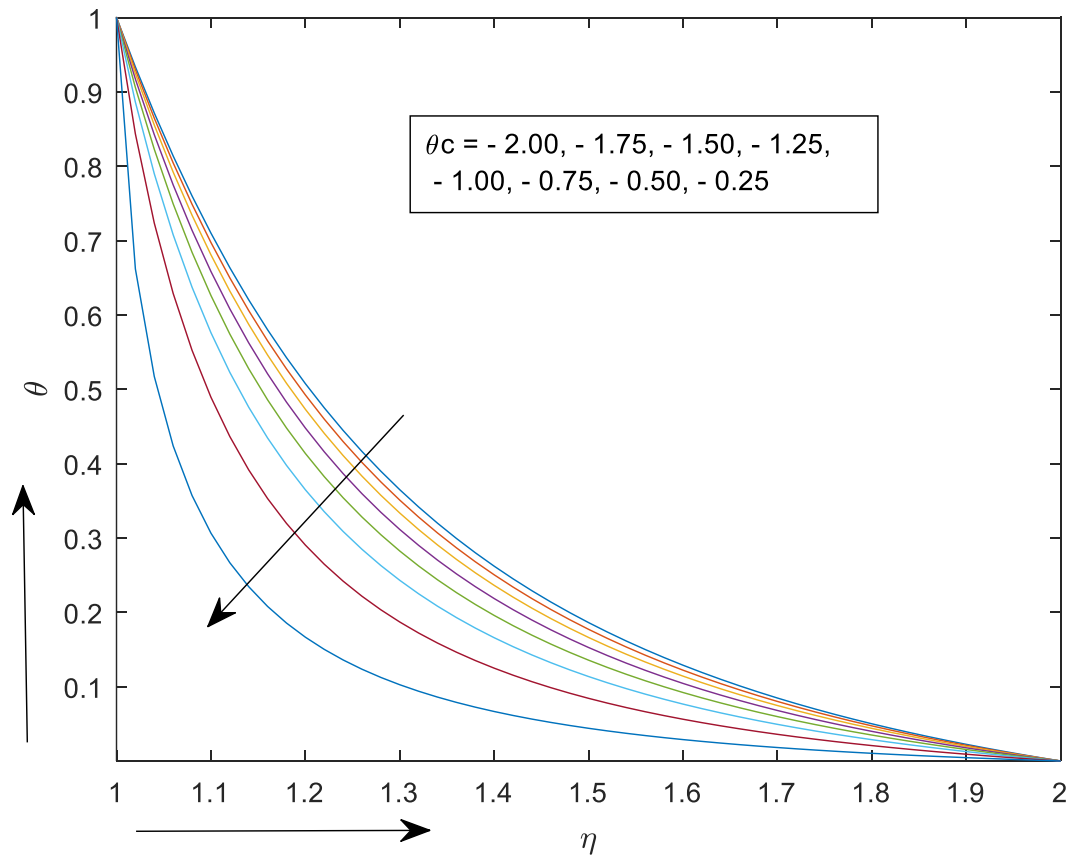

Figure 8. Temp. profile for different values of $\theta_{c}$ 
International Journal of Mathematical, Engineering and Management Sciences

Vol. 4, No. 3, 745-760, 2019

https://dx.doi.org/10.33889/IJMEMS.2019.4.3-058

Angular velocity profile for various parameters is shown from the Figures 9 to 12. It is observed that angular velocity increases with micropolar parameter $C_{3}$ while it decreases for the increase of the micropolar parameter $C_{2}$. Angular velocity decrease first for the increase of the viscosity parameter $\theta_{r}$ and thermal conductivity parameter $\theta_{c}$ but after some time it behave opposite.

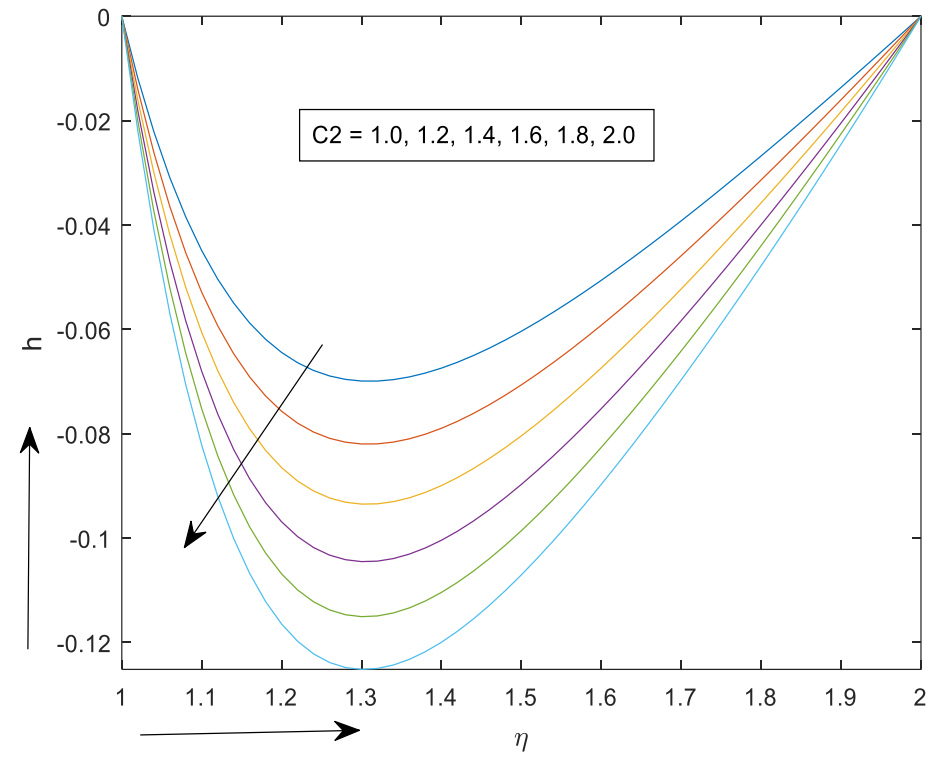

Figure 9. Angular velocity profile for different values of $C_{2}$

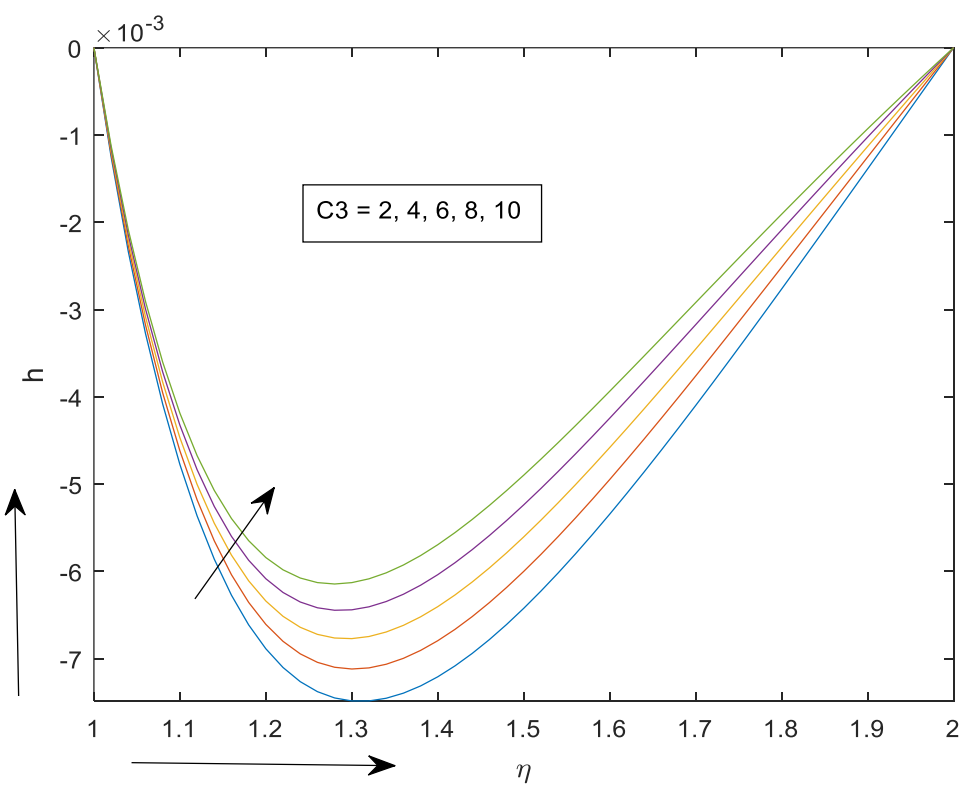

Figure 10. Angular velocity profile for different values of $C_{3}$ 
International Journal of Mathematical, Engineering and Management Sciences

Vol. 4, No. 3, 745-760, 2019

https://dx.doi.org/10.33889/IJMEMS.2019.4.3-058

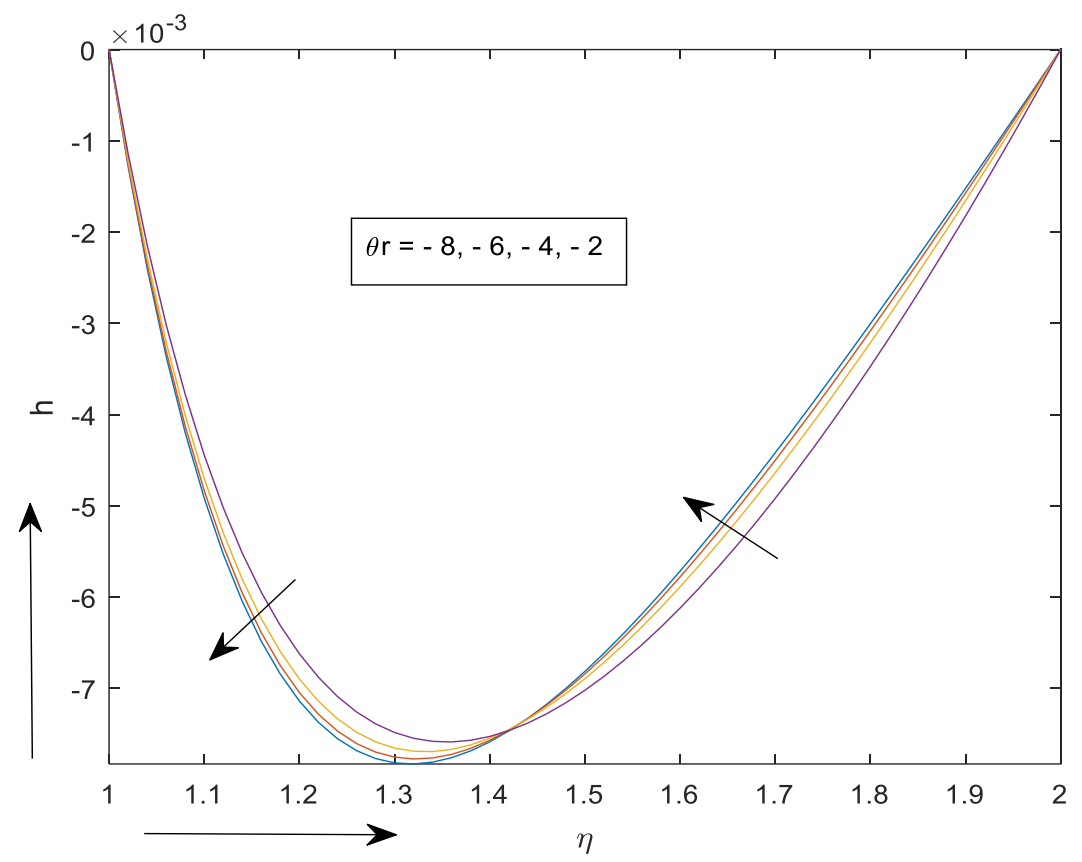

Figure 11. Angular velocity profile for different values of $\theta_{r}$

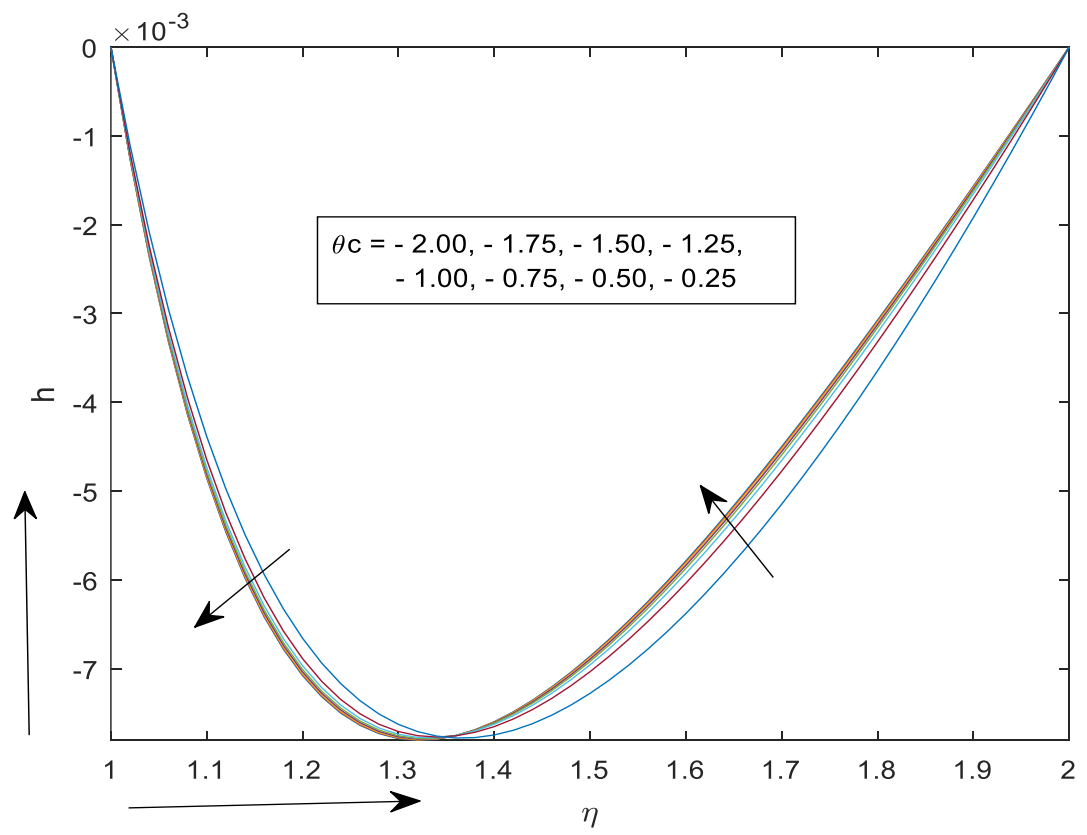

Figure 12. Angular velocity profile for different values of $\theta_{c}$ 
International Journal of Mathematical, Engineering and Management Sciences

Vol. 4, No. 3, 745-760, 2019

https://dx.doi.org/10.33889/IJMEMS.2019.4.3-058

Tables 1-4 give the missing values and physical quantities for different combination of parameters. These are self explanatory. The values of different parameters are taken as $h=.02$, $M=0.1, \theta_{c}=-12, \theta_{r}=-8, C_{1}=0.1, C_{2}=0.1, C_{3}=0.25, \operatorname{Pr}=0.72, \operatorname{Re}=10$ and $N=0.5$ unless otherwise stated.

Table 1. Missing values and physical quantities for different values of $C_{2}$ and $C_{3}$

\begin{tabular}{|c|c|c|c|c|c|c|}
\hline$C_{2}$ & $C_{3}$ & $f^{\prime \prime}(0)$ & $\theta^{\prime}(0)$ & $h^{\prime}(0)$ & $c_{f}$ & $N u$ \\
\hline \multirow{4}{*}{0.1} & 2 & -3.246924 & -0.066945 & 0.059075 & -3.210847 & 2.061819 \\
\cline { 2 - 7 } & 4 & -3.246997 & -0.065215 & 0.059076 & -3.210919 & 2.061808 \\
\cline { 2 - 7 } & 6 & -3.247067 & -0.063553 & 0.059078 & -3.210989 & 2.061798 \\
\cline { 2 - 7 } & 8 & -3.247135 & -0.061961 & 0.059079 & -3.211056 & 2.061788 \\
\cline { 2 - 7 } & 10 & -3.247201 & -0.060441 & 0.059080 & -3.211121 & 2.061778 \\
\cline { 2 - 7 } & 2 & -3.243508 & -0.132755 & 0.058991 & -3.207469 & 2.062266 \\
\cline { 2 - 7 } & 4 & -3.243650 & -0.129379 & 0.058994 & -3.207609 & 2.062245 \\
\cline { 2 - 7 } & 6 & -3.243788 & -0.126130 & 0.058997 & -3.207746 & 2.062205 \\
\cline { 2 - 7 } & 8 & -3.243921 & -0.123018 & 0.058999 & -3.207877 & 2.062205 \\
\hline \multirow{4}{*}{0.3} & 2 & -3.244049 & -0.120044 & 0.059002 & -3.208004 & 2.062186 \\
\cline { 2 - 7 } & 4 & -3.240146 & -0.197470 & 0.058909 & -3.204145 & 2.062706 \\
\cline { 2 - 7 } & 6 & -3.240354 & -0.192525 & 0.058913 & -3.204350 & 2.062675 \\
\cline { 2 - 7 } & 10 & -3.240555 & -0.187765 & 0.058917 & -3.204549 & 2.062645 \\
\cline { 2 - 7 } & 10 & -3.240750 & -0.183199 & 0.058921 & -3.204742 & 2.062616 \\
\hline
\end{tabular}

Table 2. Missing values and physical quantities for different values of $M$ and $\theta_{r}$

\begin{tabular}{|c|c|c|c|c|c|c|}
\hline$M$ & $\theta_{r}$ & $f^{\prime \prime}(0)$ & $\theta^{\prime}(0)$ & $h^{\prime}(0)$ & $c_{f}$ & $N u$ \\
\hline \multirow{4}{*}{1} & -10 & -3.434975 & -0.071656 & 0.051861 & -3.466203 & 2.034675 \\
\cline { 2 - 7 } & -8 & -3.376491 & -0.070715 & 0.060261 & -3.338974 & 2.042987 \\
\cline { 2 - 7 } & -6 & -3.288732 & -0.069318 & 0.071344 & -3.147787 & 2.055338 \\
\cline { 2 - 7 } & -4 & -3.142157 & -0.067028 & 0.085099 & -2.827941 & 2.075630 \\
\hline \multirow{4}{*}{2} & -10 & -3.602458 & -0.074519 & 0.053224 & -3.635208 & 2.009916 \\
\cline { 2 - 7 } & -8 & -3.539142 & -0.073480 & 0.061894 & -3.499819 & 2.019102 \\
\cline { 2 - 7 } & -6 & -3.444552 & -0.071946 & 0.073393 & -3.296929 & 2.032686 \\
\cline { 2 - 7 } & -4 & -3.287644 & -0.069449 & 0.087905 & -2.958880 & 2.054837 \\
\cline { 2 - 7 } & -10 & -3.796944 & -0.077852 & 0.054963 & -3.831462 & 1.980765 \\
\cline { 2 - 7 } & -8 & -3.727236 & -0.076683 & 0.063956 & -3.685823 & 1.991119 \\
\cline { 2 - 7 } & -6 & -3.623726 & -0.074968 & 0.075944 & -3.468424 & 2.006326 \\
\hline
\end{tabular}


International Journal of Mathematical, Engineering and Management Sciences

Vol. 4, No. 3, 745-760, 2019

https://dx.doi.org/10.33889/IJMEMS.2019.4.3-058

Table 3. Missing values and physical quantities for different values of $M$ and $\theta_{c}$

\begin{tabular}{|c|c|c|c|c|c|c|}
\hline$M$ & $\theta_{c}$ & $f^{\prime \prime}(0)$ & $\theta^{\prime}(0)$ & $h^{\prime}(0)$ & $c_{f}$ & $N u$ \\
\hline \multirow{4}{*}{1} & -2.00 & -3.286272 & -0.069508 & 0.064098 & -3.249758 & 2.173850 \\
\cline { 2 - 7 } & -1.75 & -3.270463 & -0.069304 & 0.064467 & -3.234125 & 2.194465 \\
\cline { 2 - 7 } & -1.50 & -3.249276 & -0.069033 & 0.064868 & -3.213173 & 2.221343 \\
\cline { 2 - 7 } & -1.25 & -3.219442 & -0.068657 & 0.065280 & -3.183670 & 2.257980 \\
\cline { 2 - 7 } & -1.00 & -3.174402 & -0.068099 & 0.065635 & -3.139131 & 2.311228 \\
\hline \multirow{4}{*}{2} & -2.00 & -3.439084 & -0.072112 & 0.064540 & -3.400872 & 2.149683 \\
\cline { 2 - 7 } & -1.75 & -3.421637 & -0.071882 & 0.064779 & -3.383619 & 2.170330 \\
\cline { 2 - 7 } & -1.50 & -3.398290 & -0.071576 & 0.065028 & -3.360531 & 2.197274 \\
\cline { 2 - 7 } & -1.25 & -3.365477 & -0.071153 & 0.065261 & -3.328083 & 2.234032 \\
\cline { 2 - 7 } & -1.00 & -3.316070 & -0.070526 & 0.065401 & -3.279225 & 2.287505 \\
\hline \multirow{4}{*}{3} & -2.00 & -3.614414 & -0.075099 & 0.065214 & -3.574253 & 2.121541 \\
\cline { 2 - 7 } & -1.75 & -3.594869 & -0.074834 & 0.065304 & -3.554926 & 2.142259 \\
\cline { 2 - 7 } & -1.50 & -3.568766 & -0.074483 & 0.065378 & -3.529113 & 2.169322 \\
\cline { 2 - 7 } & -1.25 & -3.532174 & -0.073998 & 0.065404 & -3.492927 & 2.206284 \\
\cline { 2 - 7 } & -1.00 & -3.477265 & -0.073283 & 0.065299 & -3.438629 & 2.260112 \\
\hline
\end{tabular}

Table 4. Missing values and physical quantities for different values of $\theta_{r}$ and $\theta_{c}$

\begin{tabular}{|c|c|c|c|c|c|c|}
\hline$\theta_{r}$ & $\theta_{c}$ & $f^{\prime \prime}(0)$ & $\theta^{\prime}(0)$ & $h^{\prime}(0)$ & $c_{f}$ & $N u$ \\
\hline \multirow{5}{*}{-10} & -2.00 & -3.230471 & -0.068434 & 0.054885 & -3.259839 & 2.183194 \\
\cline { 2 - 7 } & -1.75 & -3.217859 & -0.068272 & 0.055297 & -3.247112 & 2.203450 \\
\cline { 2 - 7 } & -1.50 & -3.200910 & -0.068057 & 0.055761 & -3.230010 & 2.229839 \\
\cline { 2 - 7 } & -1.25 & -3.176956 & -0.067756 & 0.056271 & -3.205838 & 2.265774 \\
\cline { 2 - 7 } & -1.00 & -3.140601 & -0.067308 & 0.056792 & -3.169152 & 2.317948 \\
\hline \multirow{5}{*}{-8} & -2.00 & -3.163817 & -0.067418 & 0.063861 & -3.128663 & 2.192989 \\
\cline { 2 - 7 } & -1.75 & -3.149213 & -0.067233 & 0.064334 & -3.114222 & 2.213593 \\
\cline { 2 - 7 } & -1.50 & -3.129620 & -0.066987 & 0.064857 & -3.094847 & 2.240441 \\
\cline { 2 - 7 } & -1.25 & -3.101993 & -0.066644 & 0.065415 & -3.067526 & 2.277010 \\
\cline { 2 - 7 } & -1.00 & -3.060205 & -0.066135 & 0.065944 & -3.026202 & 2.330124 \\
\hline \multirow{5}{*}{-6} & -2.00 & -3.065193 & -0.065930 & 0.075859 & -2.933828 & 2.207325 \\
\cline { 2 - 7 } & -1.75 & -3.047918 & -0.065714 & 0.076422 & -2.917293 & 2.228393 \\
\cline { 2 - 7 } & -1.50 & -3.024796 & -0.065429 & 0.077029 & -2.895162 & 2.255851 \\
\cline { 2 - 7 } & -1.25 & -2.992298 & -0.065033 & 0.077645 & -2.864057 & 2.293258 \\
\cline { 2 - 7 } & -1.00 & -2.943382 & -0.064449 & 0.078159 & -2.817238 & 2.347597 \\
\hline
\end{tabular}


International Journal of Mathematical, Engineering and Management Sciences

Vol. 4, No. 3, 745-760, 2019

https://dx.doi.org/10.33889/IJMEMS.2019.4.3-058

Tables 5-7 show the comparison of missing values with the original work for different parameters.

Table 5. Comparison of missing values with original paper for different $C_{1}$

\begin{tabular}{|c|c|c|c|c|c|c|}
\hline \multirow{2}{*}{$C_{1}$} & \multicolumn{2}{|c|}{$f^{\prime \prime}(0)$} & \multicolumn{2}{c|}{$\theta^{\prime}(0)$} & \multicolumn{2}{c|}{$h^{\prime}(0)$} \\
\cline { 2 - 7 } & $\begin{array}{c}\text { Nazir A et } \\
\text { al.(2015) }\end{array}$ & $\begin{array}{c}\text { Present } \\
\text { result }\end{array}$ & $\begin{array}{c}\text { Nazir A et } \\
\text { al.(2015) }\end{array}$ & $\begin{array}{c}\text { Present } \\
\text { result }\end{array}$ & $\begin{array}{c}\text { Nazir A et } \\
\text { al.(2015) }\end{array}$ & $\begin{array}{c}\text { Present } \\
\text { result }\end{array}$ \\
\hline 0.1 & -1.247970 & -3.246859 & -0.769629 & -0.068510 & -2.061382 & 0.059074 \\
\hline 0.2 & -1.227005 & -3.389545 & -0.768485 & -0.070885 & -2.062061 & 0.058251 \\
\hline 0.3 & -1.205356 & -3.519781 & -0.767433 & -0.073086 & -2.062050 & 0.057355 \\
\hline 0.4 & -1.182944 & -3.638733 & -0.766513 & -0.075120 & -2.061155 & 0.056399 \\
\hline 0.5 & -1.159920 & -3.747455 & -0.765673 & -0.076998 & -2.059687 & 0.055397 \\
\hline
\end{tabular}

Table 6. Comparison of missing values with original paper for different $C_{2}$

\begin{tabular}{|c|c|c|c|c|c|c|}
\hline \multirow{2}{*}{$C_{2}$} & \multicolumn{2}{|c|}{$f^{\prime \prime}(0)$} & \multicolumn{2}{c|}{$\theta^{\prime}(0)$} & \multicolumn{2}{c|}{$h^{\prime}(0)$} \\
\cline { 2 - 7 } & $\begin{array}{c}\text { Nazir A et } \\
\text { al.(2015) }\end{array}$ & $\begin{array}{c}\text { Present } \\
\text { result }\end{array}$ & $\begin{array}{c}\text { Nazir A et } \\
\text { al.(2015) }\end{array}$ & $\begin{array}{c}\text { Present } \\
\text { result }\end{array}$ & $\begin{array}{c}\text { Nazir A et } \\
\text { al.(2015) }\end{array}$ & $\begin{array}{c}\text { Present } \\
\text { result }\end{array}$ \\
\hline 0.1 & -1.264106 & -3.246859 & -0.770618 & -0.068510 & -0.084340 & 0.059074 \\
\hline 0.2 & -1.260262 & -3.243382 & -0.770694 & -0.135806 & -0.154769 & 0.058989 \\
\hline 0.3 & -1.257286 & -3.239961 & -0.770730 & -0.201935 & -0.216289 & 0.058906 \\
\hline 0.4 & -1.254883 & -3.236595 & -0.770741 & -0.266938 & -0.271619 & 0.058825 \\
\hline 0.5 & -1.252881 & -3.233282 & -0.770737 & -0.330855 & -0.322380 & 0.058745 \\
\hline
\end{tabular}

Table 7. Comparison of missing values with original paper for different $C_{3}$

\begin{tabular}{|c|c|c|c|c|c|c|}
\hline \multirow{2}{*}{$C_{3}$} & \multicolumn{2}{|c|}{$f^{\prime \prime}(0)$} & \multicolumn{2}{c|}{$\theta^{\prime}(0)$} & \multicolumn{2}{c|}{$h^{\prime}(0)$} \\
\cline { 2 - 7 } & $\begin{array}{c}\text { Nazir A et } \\
\text { al.(2015) }\end{array}$ & $\begin{array}{c}\text { Present } \\
\text { result }\end{array}$ & $\begin{array}{c}\text { Nazir A et } \\
\text { al.(2015) }\end{array}$ & $\begin{array}{c}\text { Present } \\
\text { result }\end{array}$ & $\begin{array}{c}\text { Nazir A et } \\
\text { al.(2015) }\end{array}$ & $\begin{array}{c}\text { Present } \\
\text { result }\end{array}$ \\
\hline 2 & -1.920134 & -3.246924 & -0.573384 & -0.066945 & -3.513939 & 0.059075 \\
\hline 4 & -1.802885 & -3.246997 & -0.605290 & -0.065215 & -3.252205 & 0.059076 \\
\hline 6 & -1.430707 & -3.247067 & -0.713413 & -0.063553 & -2.433320 & 0.059078 \\
\hline 8 & -1.213493 & -3.247135 & -0.770406 & -0.061961 & -1.954756 & 0.059079 \\
\hline 10 & -1.206251 & -3.247201 & -0.771294 & -0.060441 & -1.927442 & 0.059080 \\
\hline
\end{tabular}

\section{Conclusion}

From the above discussions, we can conclude that the variable viscosity and thermal conductivity along with other parameters have a significant effect on velocity, temperature and micro-rotation. The following conclusions can be made:

$>$ Fluid velocity increase with micropolar parameter $C_{1}$ and $C_{2}$, viscosity parameter $\theta_{r}$ and thermal conductivity parameter $\theta_{c}$. 
International Journal of Mathematical, Engineering and Management Sciences

Vol. 4, No. 3, 745-760, 2019

https://dx.doi.org/10.33889/IJMEMS.2019.4.3-058

$>$ The temperature of the fluid enhance with magnetic parameter $M$ and micropolar parameter $C_{1}$ while there is a loss of temperature with the increase of viscosity parameter $\theta_{r}$ and thermal conductivity parameter $\theta_{c}$.

$>$ The angular velocity of the fluid increase with the increase of the micropolar parameter $C_{3}$ and decrease with the increase of the micropolar parameter $C_{2}$.

$>$ Angular velocity at first decrease and then increase with the increase of $\theta_{r}$ and $\theta_{c}$.

This study will be helpful to study the properties of fluid with more parameters.

\section{Conflict of Interest}

The author confirms that this article contents have no conflict of interest.

\section{Acknowledgement}

The author would like to express his sincere thanks to the referees for their valuable suggestions towards the improvement of the paper.

\section{References}

Asia, Y., Kashif, A., \& Muhammad, A. (2016). MHD unsteady flow and heat transfer of micropolar fluid through porous channel with expanding or contracting walls. Journal of Applied Fluid Mechanics, 9(4), 1807-1817.

Baruah, I., \& Hazarika, G.C. (2017). Effects of variable viscosity and thermal conductivity on unsteady micropolar fluid about a permeable cylinder under moving boundaries, The International Journal of Engineering and Science, 6(9), 33-41.

Eringen, A.C. (1964). Simple micro fluids. International Journal of Engineering Science, 2(2), $205-217$.

Eringen, A.C. (1966). Theory of micropolar fluids. Journal of Mathematics and Mechanics, 16(1), 1-18.

Eringen, A.C. (1972). Theory of micropolar fluids. Journal of Mathematical Analysis and Applications, 38(2), 480-496.

Guram, G. S., \& Smith, A. C. (1980). Stagnation flows of micropolar fluids with strong and weak interactions. Computers \& Mathematics with Applications, 6(2), 213-233.

Hayat T, Hussain Z, Alsaedi A, Farooq M (2016) Magnetohydrodynamic Flow by a Stretching Cylinder with Newtonian Heating and HomogeneousHeterogeneous Reactions. PLoS ONE 11(6): e0156955. doi:10.1371/journal.pone.0156955.

Hazarika, G.C. \& Hazarika, S. (2015). Effects of variable viscosity and thermal conductivity on magnetohydrodynamics mixed convective flow over a stretching surface with radiation, International Journal of Scientific Research Engineering \& Technology, 4(7), 809-815.

Hazarika, G.C., \& Phukan, B. (2016). Effects of variable viscosity and thermal conductivity on magnetohydrodynamic free convection flow of a micropolar fluid past a stretching plate through porous medium with radiation, heat generation, and Joule dissipation. Turkish Journal of Physics, 40(1), 40-51.

Ishak, A., Nazar, R., \& Pop, I. (2008). Heat transfer over a stretching surface with variable heat flux in micropolar fluids. Physics Letters A, 372(5), 559-561. 
International Journal of Mathematical, Engineering and Management Sciences

Vol. 4, No. 3, 745-760, 2019

https://dx.doi.org/10.33889/IJMEMS.2019.4.3-058

Khound, P.K. \& Hazarika, G.C. (2000). The effect of variable viscosity and thermal conductivity on liquid film on an unsteady stretching surface. Proceeding of $46^{\text {th }}$ Annual Technical Session, Association of Science Society, pp. 47-56.

Lai, F.C., \& Kulacki, F.A. (1990). The effect of variable viscosity on convective heat and mass transfer along a vertical surface in saturated porous medium. International Journal of Heat and Mass Transfer, 33(5), 1028-1031.

Mahmoud, M.A.A., \& Waheed, S.E. (2012). MHD flow and heat transfer of a micropolar fluid over a stretching surface with heat generation (absorption) and slip velocity. Journal of Egyptian Mathematical Society, 20(1), 20-27.

Nazir, A., \& Shafique, M. (2015). Numerical study of micropolar fluids flow due to a stretching cylinder by SOR iterative procedure. Universal Journal of Computational Mathematics, 3(1), 8-13.

Peddieson, J. \& McNitt, R.P. (1970), Boundary layer theory for a micropolar fluid, A.C. Eringen(Ed.), Recent Advances in Engineering Science, 5/I, Gordon \& Breach, London, pp, 405-426.

Sheri, S.R., \& Shamshuddin, M.D. (2015). Heat and mass transfer on the MHD flow of micropolar fluid in the presence of viscous dissipation and chemical reaction. Procedia Engineering, 127, 885-892.

Slayi, S.A., Idriss, H.F., \& Ashmawy, E.A. (2016). Time dependent slip flow of a micropolar fluid between two parallel plates through state space approach. Global Journal of Pure and Applied Mechanics, 12(2), 1709-1722.

Vajravelu, K., \& Hadjinicalaou, A. (1997). Convective heat transfer in an electrically conducting fluid at a stretching surface in uniform free stream, International Journal of Engineering and Science, 35(1213), 1237-1244. 